

\title{
Un spectromètre à haute résolution pour la diffusion d'électrons
}

\author{
J.B. Bellicard, Ph. Leconte
}

\section{To cite this version:}

J.B. Bellicard, Ph. Leconte. Un spectromètre à haute résolution pour la diffusion d'électrons. Revue de Physique Appliquée, 1969, 4 (2), pp.207-208. 10.1051/rphysap:0196900402020700 . jpa-00243221

\section{HAL Id: jpa-00243221 https://hal.science/jpa-00243221}

Submitted on 1 Jan 1969

HAL is a multi-disciplinary open access archive for the deposit and dissemination of scientific research documents, whether they are published or not. The documents may come from teaching and research institutions in France or abroad, or from public or private research centers.
L'archive ouverte pluridisciplinaire HAL, est destinée au dépôt et à la diffusion de documents scientifiques de niveau recherche, publiés ou non, émanant des établissements d'enseignement et de recherche français ou étrangers, des laboratoires publics ou privés. 


\title{
UN SPECTROMÈTRE A HAUTE RÉSOLUTION POUR LA DIFFUSION D'ÉLEGTRONS
}

\author{
J. B. BELLICARD et Ph. LECONTE,
}

Service de Physique Nucléaire à Haute Énergie, C.E.N., Saclay.

Résumé. - Nous exposons l'étude qui nous a conduits à choisir les caractéristiques d'un spectromètre à haute résolution, bon angle solide et assez grande acceptance en énergie.

Abstract. - We outline the study which has enabled us to choose the characteristics of a high resolution, good solid angle spectrometer and fairly large energy acceptance.

I. Résolution expérimentale. - La résolution de l'expérience de diffusion $\left(e, e^{\prime}\right)$, c'est-à-dire la possibilité de séparer deux phénomènes de diffusion se produisant à des énergies voisines, dépend de nombreux facteurs parmi lesquels on doit noter : la résolution du système de transport de faisceau, les dimensions de

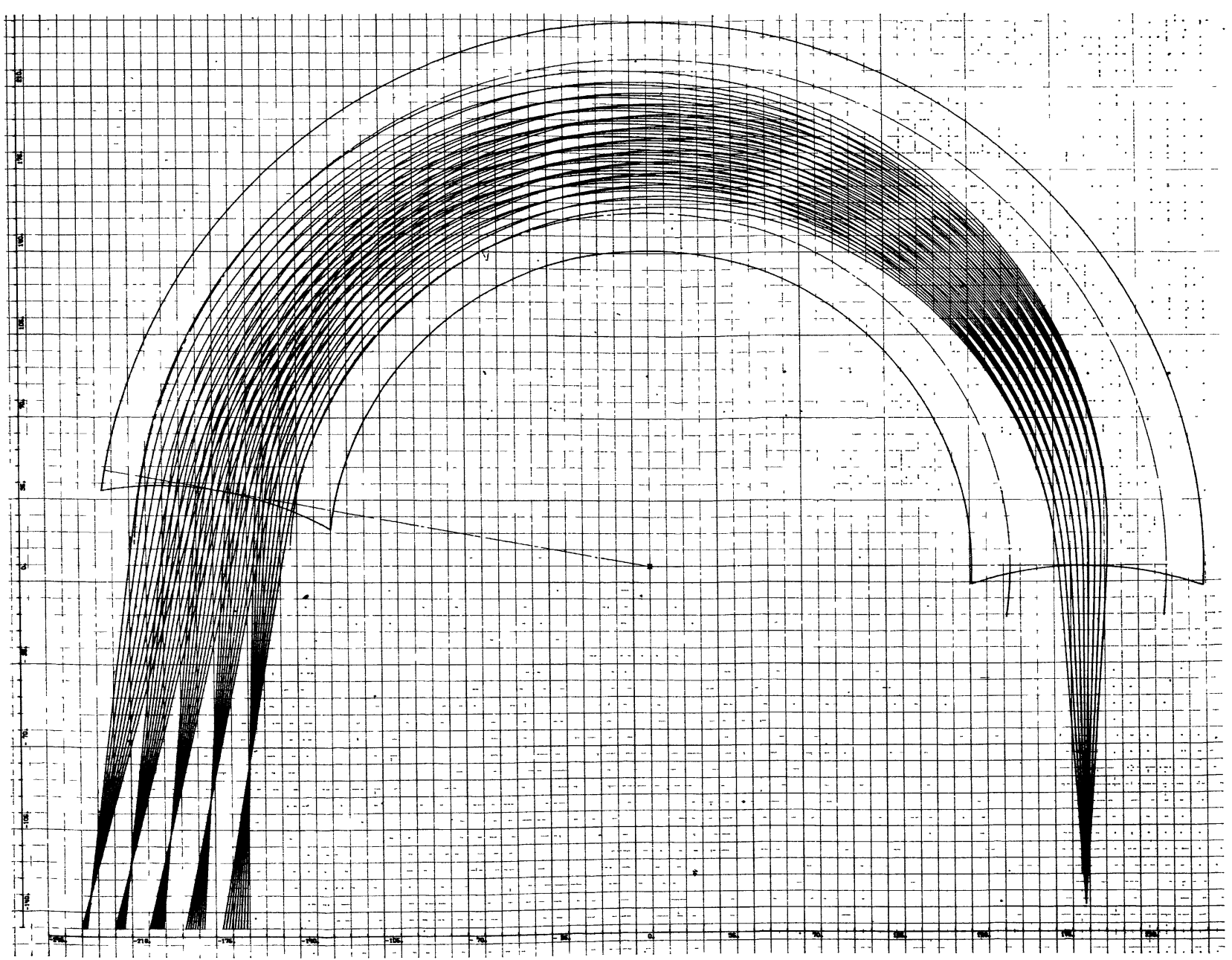

FIG. 1. - Les trajectoires dans le spectromètre : angle solide 5,6 msr; énergie - 4\%, - $2 \%, 0 \%, 2 \%, 4 \%$. 
l'impact du faisceau sur la cible, la résolution intrinsèque du spectromètre, la dimension finie des détecteurs dans le plan focal, l'épaisseur de la cible, la stabilité du faisceau en position, la stabilité des champs magnétiques, les effets dus à la cinématique.

Les conditions de l'accélérateur linéaire de Saclay ont permis de prévoir un système de transport donnant une résolution limite de $10^{-4}$ et un impact sur la cible de $1 \mathrm{~mm} \times 4 \mathrm{~mm}$. En choisissant un spectromètre de grand rayon $(1,80 \mathrm{~m})$, on peut espérer limiter l'effet des deux premiers facteurs à $1,4 \times 10^{-4}$. Il faut par conséquent construire un spectromètre qui ait une résolution de l'ordre de $10^{-4}$ avec un angle solide aussi grand que possible.

II. Résolution intrinsèque du spectromètre. - On cherche une focalisation radiale ultra-fine et on dispose pour cela des paramètres suivants $: i$ ) profil radial du champ; ii) rayon de courbure des faces; iii) angle total de déviation. Avec ces paramètres, on peut toujours obtenir une correction des aberrations au second ordre pour les termes dépendant de l'ouverture angulaire en appliquant une relation d'Ikegami [1]. Un cas intéressant est celui de l'angle magique découvert par Sakai [2] et appliqué également par S. Penner [3]. La solution choisie pour le spectromètre de Saclay conserve l'angle de $169^{\circ} 7$ mais est améliorée pour obtenir une bonne conservation des propriétés de focalisation transversale sur tout le plan focal. D'autre part, nous avons tenu à rendre l'aimant améliorable, toujours en jouant sur la forme des faces d'entrée et de sortie qui sont démontables et peuvent être corrigées par approximations successives. Les calculs précis sur ces formes de faces ont été conduits à l'aide du programme Orbite [4] qui permet, par une intégration pas à pas, de déterminer des trajectoires dans n'importe quelle configuration de champ et, par conséquent, de rendre compte de variations de rayon de courbure des faces.

III. Maquettes. - Une maquette de l'aimant a été réalisée par la société Oerlikon. Elle a été construite

avec une face courbe et une face droite de façon à disposer de cartes de champ pour toutes les formes de faces qui doivent permettre d'atteindre la meilleure résolution. On a pu contrôler de cette façon les effets de dimension et de forme du circuit magnétique, les effets d'entrefer filtre, la forme des profils antisaturants et leur efficacité, la courbure de la face magnétique, la longueur optique.

La résolution intrinsèque obtenue en introduisant ces mesures dans le programme Orbite a été meilleure que $10^{-4}$ sur la partie centrale du plan focal $( \pm 2 \%)$. Une adaptation sur l'aimant définitif sera encore nécessaire pour assurer cette propriété sur tout le plan focal. Les calculs ont montré que cette adaptation est toujours possible.

IV. Caractéristiques. - L'aimant est en cours de réalisation par la société Oerlikon avec les caractéristiques suivantes :

Impulsion maximale analysée : Induction maximale correspondante :

Rayon moyen :

Angle total de déviation

Face d'entrée et face de sortie orthogonales au rayon moyen

Face d'entrée et face de sortie concave : rayon de courbure :

Distance objet $=$ distance image :

Indices de champ :

Entrefer au rayon de référence :

Précision sur l'entrefer sur toute la surface :

Acceptance en impulsion :

Angle solide :

Résolution :

Dispersion :

Poids :

Année de mise en service :

Puissance de l'alimentation électrique :
$900 \mathrm{MeV} / \mathrm{c}$

1,67 tesla

$1,8 \mathrm{~m}$

$169^{\circ} 42^{\prime}$

$1,47 \mathrm{~m}$

$1,47 \mathrm{~m}$

$n=1 / 2, \quad \beta=1 / 6$

$120 \mathrm{~mm}$

$\pm 0,20 \mathrm{~mm}$

$\pm 5 \%$

de 2,5 à $5,6 \mathrm{msr}$

$2 \times 10^{-4}$

$7,2 \mathrm{~mm} / \%$

$184 \mathrm{t}$

fin 1970

$280 \mathrm{~kW}$.

\section{BIBLIOGRAPHIE}

[1] Ikegami (H.), Rev. Sci. Instr., 1958, 29, 943.

[2] Sakai (M.), Nucl. Instr. Methods, 1960, 8, 61.

[3] Penner (S.) et Lightbody (J. W.), Proc. Int. Symp. on Magnet Technology of Stanford.

[4] Mougey (J.) et LEconTe (P.), ce colloque. 